\title{
Propuesta didáctica para la comprensión crítica en la Universidad La Gran Colombia*
}

\author{
JOSÉ LEONARDO SERNA DÍAZ" \\ leonardo.serna@ugc.edu.co \\ JORGE ENRIQUE DÍAZ CORTÉS \\ jorge.diaz@ugc.edu.co
}

Forma de citar este artículo: Serna Díaz, J., \& Díaz Cortés, J. (2014). Propuesta didáctica para la comprensión crítica en la Universidad La Gran Colombia. Cuadernos de Lingüística Hispánica, 25, 165-180. Tunja: Uptc.

\footnotetext{
* Artículo de investigación. Hace parte de las contribuciones y reflexiones que realiza actualmente el grupo de investigación: "Educación y Pedagogía" desde la línea de investigación en pensamiento socio-crítico en la construcción significativa y solidaria del conocimiento de la Universidad La Gran Colombia (UGC).

** Coordinador y docente de la Licenciatura en Lingüística y Literatura de la Universidad La Gran Colombia.

**** Docente e investigador de la Licenciatura en Lingüística y Literatura de la Universidad La Gran Colombia y docente de la Secretaría de Educación Distrital de Bogotá.
} 


\section{Resumen}

El presente artículo muestra parte de un trabajo investigativo que actualmente se adelanta sobre el desarrollo de la comprensión crítica en los estudiantes de la Licenciatura en Lingüística y Literatura y de primer semestre de otros programas académicos de la Universidad La Gran Colombia. Su objetivo es proponer estrategias didácticas para realizar una lectura crítica. Por esta razón, se expone el recorrido teórico-metodológico que se ha realizado para crear dichas prácticas; en la primera parte, se revisa de modo introductorio la problemática que gira alrededor de la comprensión lectora; en segundo lugar, se presenta el marco referencial, que expone distintas perspectivas de la lectura como concepto científico. Acto seguido, se relaciona un aspecto fundamental para el progreso de la indagación que tiene que ver con la incorporación del Análisis Crítico del Discurso y su relación adyacente con el conocimiento de las realidades presentes en los textos. Finalmente, se registran algunos avances metodológicos sobre la posibilidad, proyección y necesidad de fomentar este tipo de práctica lectora en el aula universitaria.

Palabras clave: lectura crítica, Análisis Crítico del Discurso, discurso académico, prácticas lectoras.

\section{Didactic proposal for critical comprehension at La Gran Colombia University}

\section{Abstract}

This article shows part of a research work that is currently being carried out, about the development of critical comprehension in students of the Linguistics and Literature Bachelors Program, as well as with first semester students of other academic programs, at La Gran Colombia University. The objective is to propose didactic strategies for achieving critical reading. For this reason, the theoretical - methodological itinerary which has led to the creation of such practices is exposed. The first part contains an introductory review of the problem of reading comprehension; secondly, a referential framework about different perspectives on reading as a scientific concept is presented. Then, critical discourse analysis is incorporated into the study, and its adjacent relation with knowledge of the realities present in the texts. Finally, methodological advances are presented, related to the possibility, projection and need of promoting this type of reading practice at the university level.

Keywords: critical reading, critical discourse analysis, academic discourse, reading practices 


\section{Proposition didactique pour la compréhension critique dans l'Université La Gran Colombia}

\section{Résumé}

Cet article montre une partie de notre travail de recherche, réalisé en ce moment, sur le développement de la compréhension critique chez les étudiants de la Licence en Linguistique et Littérature et ceux du premier semestre d'autres programmes académiques de l'Université $L a$ Gran Colombia. L'objectif du travail est celui de proposer des stratégies didactiques pour réaliser une lecture critique. Pour cette raison, il est exposé le parcours théorico-méthodologique qui a été réalisé pour créer ces pratiques-là. Dans la première partie, on révise de manière introductoire la problématique qui tourne autour de la compréhension écrite. En deuxième lieu, on présente le cadre référentiel qui expose de diverses perspectives de la lecture en tant que concept scientifique. Tout de suite après, on relie un aspect fondamental pour le progrès de la recherche, qui a à voir avec l'incorporation de l'Analyse Critique du Discours et son rapport adjacent avec la connaissance des réalités présentes dans les textes. Finalement, on enregistre quelques avances méthodologiques sur la possibilité, projection et besoin d'encourager ce type de pratique de lecture dans la salle de classe à l'université.

Mots clés: lecture critique, Analyse Critique du Discours, discours académique, pratiques de lecture..

\section{Proposta didática para a compreensão crítica na Universidade La Gran Colombia}

\section{Resumo}

0 presente artigo mostra parte de um trabalho de pesquisa que atualmente é feito sobre 0 desenvolvimento da compreensão crítica nos estudantes da Licenciatura em Linguística e Literatura e de primeiro semestre de outros programas acadêmicos da Universidade La Gran Colombia. Seu objetivo é propor estratégias didáticas para realizar uma leitura crítica. Por esta razão, se expõe o percurso teórico-metodológico que se tem realizado para criar tais práticas; na primeira parte, se revisa de modo introdutório a problemática que gira ao redor da compreensão leitora; em segundo lugar, se apresenta o marco referencial, que expõe distintas perspectivas da leitura como conceito científico. Ato seguido se relaciona um aspecto fundamental para o progresso da indagação que tem que ver com a incorporação da Análise Crítica do Discurso e sua relação adjacente com o conhecimento das realidades presentes nos textos. Finalmente, se registram alguns avanços metodológicos sobre a possibilidade, projeção e necessidade de fomentar este tipo de prática leitora na aula universitária.

Palavras chave: leitura crítica, Análise Crítico do Discurso, discurso acadêmico, práticas leitoras. 


\section{Introducción}

Uno de los principales motivos de éxito y, a su vez, de fracaso escolar consiste en aprender a leer de manera comprensiva. Algunas personas durante toda su formación académica aprenden a desarrollar procesos de comprensión que sobrepasan el nivel literal de la lectura, haciendo de esto un ejercicio significativo que cobra sentido; sin embargo, otro gran número de personas en procesos de formación profesional presentan muchas dificultades a la hora de leer comprensivamente un texto, sobre todo cuando las características de los documentos universitarios, en su mayoría, se presentan bajo las diversas tipologías del texto académico.

Algunas de las causas por las cuales la lectura no es significativa para los estudiantes, radica en el hecho de pensar que la lectura es una práctica exclusiva de la academia, puesto que muchas veces los estudiantes leen con la finalidad de aprobar un examen 0 presentar una exposición; en esa medida, el propósito de la lectura no es otro que el de la nota, es simplemente un deber o una obligación.

De esta manera, las evidencias que sustentan estas premisas investigativas se encuentran en los resultados de las pruebas internacionales y nacionales sobre la comprensión lectora. Dichos resultados han puesto de manifiesto serias dificultades en el dominio de la lectura. En muchos países latinoamericanos, los procesos en lectura se sitúan por debajo del promedio de los países de la OCDE (Organización para la Cooperación y el Desarrollo Económico). La prueba PISA (Programme for International Student Assessment) del año 2009, por ejemplo, mostró en los resultados de comprensión lectora que nuestro país obtuvo un puntaje de 385 , que nos ubica significativamente más bajo que la media de la OCDE y por debajo de países latinoamericanos como Brasil, que ocupó la casilla 393; México, que se ubicó en la casilla 410; Uruguay, por su parte, en la casilla 413 y Chile, en la casilla 442 en orden ascendente. Por su parte, en el 2012 los resultados de PISA muestran que solamente 3 de cada mil estudiantes pueden hacer inferencias múltiples, efectuar comparaciones y contrastes detallados y precisos, lo que nuevamente evidencia los bajos niveles en la comprensión lectora de los jóvenes colombianos.

Por su parte, los resultados de la prueba nacional Saber Once realizada por el ICFES (Instituto Colombiano para la Evaluación de la Educación) y aplicada a estudiantes de grado undécimo, como requisito de ingreso a la educación superior, muestran que durante el período comprendido entre los años 2008 y 2010 la media nacional en lenguaje ha tenido una constante en cuanto al promedio obtenido, oscila entre el $45.44 \%$ y el $46.14 \%$ de rendimiento o eficiencia a la hora de contestar la prueba, lo que indica que la media nacional posee un promedio mínimo, con ciertos casos aislados de satisfacción ubicados 
en los colegios rurales y urbanos de la capital del país, a diferencia de algunos colegios privados que alcanzan niveles satisfactorios y avanzados.

Los anteriores resultados indican una problemática bastante seria, referida a los aprendizajes de los estudiantes en relación con la comprensión lectora y sobre todo en lo concerniente a nuestro estudio apoyado en la lectura crítica. La mayoría de los estudiantes no desarrollan tareas complejas en los textos que leen, se ubican en los niveles de desempeño básico, donde las tareas exigen localizar e identificar informaciones explícitamente enunciadas en el texto. Las cifras muestran que una proporción significativa de los jóvenes de nuestro país no cuentan con las estrategias requeridas para participar activamente en la sociedad. Es decir, los estudiantes no establecen una relación entre lo que aprenden en la escuela y el uso social del conocimiento; por tanto, es primordial desarrollar una serie de estrategias que lleven al lector de un nivel novato a un nivel de experto.

De esta manera, la lectura es concebida en este estudio como una práctica social enfocada al desarrollo de estrategias que permitan crear procesos reflexivos y críticos sobre el entorno y sobre el saber que se adquiere durante la formación profesional. Dentro de dichas estrategias se resalta el conocimiento de las condiciones de producción de los textos, la relación con la realidad nacional y mundial, la identificación de las ideologías presentes en los discursos, entre otras. Así, se hace partícipe a los estudiantes de los desarrollos teóricos de su disciplina específicamente con estudiantes de primer semestre de los programas de Arquitectura, Administración de empresas, Contaduría y de diferentes semestres de la Licenciatura en Linguística y Literatura.

\section{Reflexión: entre teoría y práctica}

En el estudio e indagación que se ha desarrollado en torno a la problemática de la lectura crítica, tema propuesto como eje fundamental para el desarrollo de la investigación, se ha evidenciado que existe un criterio de organización en lo que se refiere a la teoría de la lectura y, en especial, a la relación que teje con la pedagogía de la lengua.

En tal sentido, al investigar el concepto de lectura crítica contemporánea se hace necesario entender, qué significa la lectura y sobre todo el sentido que se otorga a la noción de comprensión crítica en este momento socio-histórico. Para la aproximación a los referentes conceptuales de la lectura crítica, fue necesario realizar una indagación en los estudios que abarcan disciplinas científicas como la Lingüística, la Psico-cognición y los Estudios socio-culturales, que brindaron el soporte para los diferentes modelos de enseñanza-aprendizaje de la lectura. 
En la actualidad, saber leer deja de ser un privilegio para transformarse en una necesidad o un requisito para comprender y circular en el mundo, "para participar de la cultura y del conocimiento" (Santiago, 2010, p. 26). Aprender a leer implica adquirir y desarrollar conocimientos socioculturales sobre diferentes discursos; en este caso, nuestro proyecto busca generar el desarrollo de dichos procesos para formar lectores críticos desde el análisis de diferentes discursos académicos y mediáticos.

La lectura, a lo largo de la historia reciente, ha sido reconocida como una práctica social y cultural, por ende "la comprensión proviene de la comunidad de hablantes; el significado nace de la cultura que comparten el autor y el lector. Este es el fundamento del enfoque teórico sociocultural" (Cassany, 2006. 13). Sin embargo, para entender este enfoque es necesario hacer una aproximación conceptual sobre las inter-disciplinas que han dinamizado la lectura como proceso.

\subsection{Los Estudios del Lenguaje}

Los estudios científicos sobre el lenguaje se consolidan en la primera mitad del siglo XX, "solo desde bien entrado el siglo XX, con los desarrollos ejemplares de la lingüística estructural saussureana, se establecieron las condiciones epistemológicas para que la lectura fuera una práctica intelectual esclarecida con el rigor y los predicamentos de la ciencia" (Moreno et al., 2010, p. 147). De esta forma, se generó en la pedagogía del lenguaje un modelo de lectura Bottom up, o ascendente, en el cual la comprensión se concibe como una serie de pasos que atraviesan el entendimiento, en una secuencia directa que va desde la letra, hasta el texto. De tal manera, la comprensión y el significado del texto se encuentra por fuera de la mente del lector y dentro del texto leído.

El enfoque lingüístico entiende la lectura como un proceso perceptivo y secuencial. En esa medida, el signo es quien genera el proceso de significación y el receptor requiere solo de los conocimientos básicos del código escrito para realizar un proceso que apenas va un poco más allá de la decodificación. El lector que no supera el significado textual se le conoce como: "analfabeto funcional, quien no puede comprender la prosa, aunque pueda oralizarla en voz alta” (Cassany, 2006, p. 21); se puede verbalizar el código escrito sin importar si se comprende o no, desde este enfoque lo que interesa es que el estudiante vocalice bien y tenga buena dicción, sin importar el nivel de comprensión que obtenga del discurso que lee.

El enfoque lingüístico proporciona valiosos aportes para el desarrollo de la comprensión crítica, provee la esfera académica para la relación entre los procesos textuales y los procesos mentales presentes en los discursos. Para el estudio es fundamental que el estudiante conozca qué es una categoría gramatical, un modo verbal o una voz 
pasiva, por ejemplo; pero lo importante no es solo conocer los aspectos formales, sino entender que al rastrear este tipo de elementos se puede hacer una mejor comprensión del discurso. Además, gracias a los desarrollos teóricos de la lingüística, la lectura empieza a concebirse como objeto de estudio para las ciencias humanas.

\subsection{Los estudios psico-cognitivos}

Con el desarrollo científico de la psicología cognitiva surgen cambios paradigmáticos, aproximadamente en la década de los sesenta y setenta aparece y se desarrolla gran parte de los estudios con un interés marcado por la comprensión y el uso práctico de los conocimientos, lo que "implicó la impugnación de la posición empirista y descriptiva de Bloomfield y sus sucesores, para darle a la lingüística una orientación cognitiva" (Pardo, 2008, p. 13), desde este enfoque, se concibe la lectura como un proceso dinámico, interactivo y transaccional de carácter cognitivo.

Un aspecto que se ha de tener en cuenta para diferenciar el enfoque lingüístico del psico-cognitivo, es la relación entre el texto y el lector; desde los estudios cognitivos de la lectura, los participantes desarrollan un papel activo en el proceso de comprensión, es decir, se tiene en cuenta que el significado se da también en la mente del lector, pues se construye gracias a la interacción con el texto y sus experiencias, en contraste con el enfoque lingüístico.

Las investigaciones desde este campo de indagación se dedican al estudio de los procesos cognitivos, el papel de la mente y cómo ella misma permite el desarrollo de la comprensión. De tal manera, que la comprensión

implica un esfuerzo intelectual que busca captar el significado global del texto y para esto se realiza una serie de representaciones organizadas del texto leído. La lectura desde este enfoque es el proceso que permite revisar y usar las claves lingüísticas" (Santiago, 2005, p. 27).

Entender la lectura como un proceso cognitivo implica poner en marcha una serie de procesos, entre otros: abstraer, analizar, sintetizar, predecir, comparar y asociar, con el fin de llegar a un mejor nivel de comprensión ante lo que se lee.

Precisamente, en función de mejorar los procesos cognitivos para desarrollar una comprensión crítica en los estudiantes, la investigación plantea también la necesidad de recurrir a estrategias meta-cognitivas, las cuales aportan de manera significativa en el proceso de comprensión. "El conocimiento metacognitivo permite, entonces, que se pueda adelantar la supervisión y la regulación de los distintos aspectos que intervienen en el desarrollo de una tarea cognitiva" (Santiago; 2005, p. 45), lo que le permite al estudiante 
desarrollar un tipo de conocimiento que le da la posibilidad de hacer un ejercicio de reflexión sobre su aprendizaje y sobre la actividad cognitiva que se le plantea y, con ello, mejorará de manera significativa sus procesos de comprensión.

\subsection{La Lectura como una práctica social}

Para entender el uso social de la lectura en este proyecto es inevitable mencionar el enfoque socio-cultural, precisamente porque este es la base de nuestro estandarte teórico y práctico. La lectura pasa de entenderse como un proceso de interacción del texto y el lector, a entenderse como una relación de interdependencia entre texto, lector y sociedad; los significados no solo se encuentran en la relación entre el lector y el texto, sino que también se encuentran en la comunidad de hablantes, en la cultura.

Una de las premisas fundamentales que se propone es que la lectura como concepto pedagógico y académico cambia, se transforma, no es igual leer hoy a lo que se hacía en las prácticas lectoras de la edad media o del siglo pasado. En Colombia, las transformaciones en el concepto permean las políticas públicas de educación y son prioridad para el desarrollo de una sociedad crítica; la lectura de hoy dirige sus esfuerzos a reflexionar, develar y mitigar las múltiples relaciones de poder, discriminación, xenofobia entre otras, que se establecen a partir de los discursos. Un objetivo que se pretende con el desarrollo de la investigación es la necesaria articulación entre la perspectiva del Análisis Crítico del Discurso (ACD) y una pedagogía de la lengua: "ya sean estas opacas o transparentes, las relaciones de dominación, discriminación, poder y control, se manifiestan a través del lenguaje" (Wodak y Meyer, 2001, p. 19) y muchos de los discursos que circulan en la sociedad académica contienen ideologías que deben ser develadas.

En ese sentido, "aprender a leer requiere no solo desarrollar los mencionados procesos cognitivos, sino también, adquirir los conocimientos socioculturales particulares de cada discurso, de cada práctica concreta de lectoescritura" (Cassany, 2006, p. 24). En esa medida, los conocimientos adquiridos en la clase de lenguaje o competencias comunicativas, asignatura transversal en la Universidad, permiten generar una reflexión constante sobre los hechos sociales que aparecen en la realidad, para determinar la autenticidad y poder asumir una posición frente a los mismos a partir de un análisis lector que trascienda los niveles literales.

Al concebir la lectura como un proceso de comprensión y, mas aún, como un proceso sociocultural, es necesario entender que la comprensión se concibe como la interacción entre el texto-autor, el lector y la sociedad, por ende la lectura no es un proceso unidireccional o interactivo entre el texto y el lector. Es decir, un lector con un mayor conocimiento de la realidad social y disciplinar va a poseer un alto nivel de comprensión 
ante una noticia o un artículo de investigación, puesto que para él, no solo es importante saber el significado de cada una de la palabras, hacer hipótesis, crear inferencias, sino interactuar con el discurso y tomar una posición frente a lo que lee. Este tipo de lectura se interesa por valorar el discurso en pro de establecer las condiciones de producción, la validez del texto, de reconocer las posibles ideologías dominantes y de participar en los efectos que causa el discurso en el ámbito social.

Desde el enfoque socio-cultural de la lectura, encontramos que los significados tienen origen social; discurso, autor y lector no están aislados. Al respecto, "tres son los conceptos que, de manera indispensable, han de figurar en todo ACD: el concepto de poder, el concepto de historia y el concepto de ideología" (Wodak y Meyer, 2001, p. 19). De esta manera, el lector experto desde la experiencia que posee del contexto, del autor y del texto reconoce que el enunciador produce el discurso desde una comunidad, desde una ideología.

Para el desarrollo de la investigación, el concepto de comprensión crítica es fundamental, puesto que delimita el tipo de comprensión que queremos desarrollar en nuestros estudiantes. Existen diversos tipos de comprensión así como existen diversos tipos de sociedades; la comprensión crítica es diferente de otros niveles de comprensión porque busca lo que está detrás de las líneas, "lo que hay detrás de las líneas es la ideología, el punto de vista, la intención y la argumentación que apunta realizar el autor" (Cassany, 2006, p. 52) y alcanzar ese nivel de comprensión implica el haber desarrollado procesos para comprender las relaciones entre el discurso público, y la sociedad en este caso.

Leer de forma crítica es estar en la capacidad de comprender los significados ocultos o implícitos de los textos y tomar una posición ante ellos; comprender críticamente implica estar en la capacidad de utilizar esos significados para crear un modelo de la situación comunicativa. De esta manera, en el enfoque sociocultural de la lectura se requiere percibir la relación entre el texto y la sociedad. En otras palabras, se busca que el uso pedagógico de la perspectiva del ACD permita despertar conciencia en los estudiantes de lo dinámico que son hoy en día los discursos.

Sin embargo, para crear un impacto real en la forma de concebir la lectura por parte de los estudiantes, es necesario delimitar cómo realizaremos el análisis desde el enfoque sociocultural, es decir nos interesa caracterizar cómo la perspectiva del ACD se convierte en una estrategia de real importancia en el desarrollo de las políticas de lectura y escritura en la universidad. 


\subsection{La perspectiva del Análisis Crítico del Discurso (ACD) como estrategia para el desarrollo de la comprensión}

La perspectiva del Análisis Crítico del Discurso permitió conocer algunas categorías de análisis para aproximar y desarrollar la comprensión crítica en los estudiantes. Teniendo en cuenta, que: "El análisis crítico del discurso como escenario de conocimiento se encuentra comprometido con dos aspectos fundamentales: la formulación de desarrollos teóricos en torno al discurso, y la reflexión crítica sobre las realidades sociales presentes en los discursos" (Pardo, 2008, p. 17). Más que convertir la perspectiva del ACD en una propuesta de carácter pedagógico, se toman las propuestas como referencia disciplinar, además se recalca que el fundamento del "ACD debería ser accesible a todas las personas" (Van Dijk, 2003, p. 145). De esta manera, la propuesta investigativa es concurrente con los objetivos fundamentales de la investigación crítica, puesto que, "el análisis de la realidad social debe poder ser compartido por otros" (Serrano, 2007, p. 506); una reflexión que suscita este estudio es la labor docente como práctica para el cambio social.

Para dar inicio a una breve introducción de la propuesta didáctica es inevitable entender que el Análisis Crítico del Discurso es una teoría transdisciplinar que está relacionada con el poder, el abuso del poder y la forma como se mantienen la desigualdad por medio de la comunicación y el uso de la lengua. Entonces, el ACD es una actitud crítica enfocada a los problemas sociales de mayor relevancia en un momento histórico y en una sociedad en particular, que pretende describir de manera adecuada los diferentes tipos de discurso y sus intenciones de dominación y desigualdad, además de evidenciar las relaciones del texto y el contexto en la dominación de las relaciones del poder discursivo.

Para comprender el abuso del poder, lo definiremos como el control que un grupo de personas privilegiado en recursos sociales (como dinero, fama o cultura) ejerce sobre otro grupo de personas con menos recursos económicos, culturales o educativos, "Los primeros tienen acceso y dominan los discursos públicos (políticos, mediáticos, educativos, científicos, legales y burocráticos) aquellos discursos que ejercen un poder sobre las mentes de aquellos menos afortunados, viviendo bajo modelos que continúan reproduciendo la desigualdad" (Van Dijk, 2004, p. 16). Los textos están inmersos en una sociedad, en una cultura particular y que por ende los discursos involucran los contextos, lo que quiere decir que una forma de controlar el discurso es controlar el contexto.

Para Van Dijk (2004), comprender un discurso significa ser capaz de crear un modelo mental y si el discurso es familiar para el lector, su comprensión será mucho más asequible, puesto que en la comprensión de los textos, los modelos de situación cumplen una función decisiva porque son fundamentales para "otorgar coherencia al 
texto". Estos modelos no son únicamente construcciones individuales, "algunas veces son construcciones colectivas que llamaremos representaciones sociales y también las podemos llamar ideología, estas tienen representaciones tanto positivas como negativas" (Van Dijk, 2004, p. 22). Las primeras tienen que ver con las tradiciones, las normas y otros aspectos de la cultura, y entre las representaciones negativas podríamos encontrar el racismo o cualquier otro tipo de discriminación. También, podemos encontrar esas ideologías en los grupos sociales que tienen afinidades en ideas de tipo político, cultural, religioso, entre otras. Así pues, la manipulación ideológica es la forma más eficiente de abusar del poder discursivo.

Desarrollar una lectura crítica implica ir más allá de la comprensión literal e inferencial, es tomar posición frente a los discursos de la cultura, para así develar las relaciones de poder y abuso del mismo. Por tanto, hacer una lectura crítica es tener una actitud para poder valorar y posicionarse frente a los discursos de la cultura.

A partir de un ejercicio de delimitación entre temáticas investigativas de los diversos enfoques del ACD, se hace necesario y preciso "optar y seleccionar para un análisis más pormenorizado de aquellas estructuras que sean relevantes para el estudio de una cuestión social. Esto nos exige al menos algunas ideas informales sobre los vínculos entre el texto y el contexto" (Van Dijk, 2003, p. 148). Por esta razón, categorías como ideología, contexto y discurso público son las seleccionadas para formalizar un análisis de carácter crítico. Por consiguiente, las categorías que se desarrollan a continuación hacen parte de la propuesta para desarrollar en los estudiantes la comprensión crítica.

\subsection{Discurso y texto en la sociedad actual}

Otro aspecto fundamental para el desarrollo de la comprensión crítica es el reconocer el género discursivo para identificar ¿Qué tipo de texto se está trabajando?, ¿a qué género discursivo pertenece? Analizar el género discursivo es importante para el desarrollo de la lectura crítica, ya que a través del reconocimiento de diversas categorías como la denominación, la función, la estructura, el estilo, la historia del género entre otros, se puede establecer una mejor relación comprensiva con el discurso.

El conocimiento del género discursivo aporta bastante en el ejercicio de comprensión, por ejemplo, con el estudio del género discursivo podemos incorporar procedimientos de voces en el discurso, identificar los estilos directos o indirectos, las mezclas de estilos, la terminología, con lo cual, nos podemos acercar de forma interactiva a la comprensión crítica. 
Es inevitable en el enfoque socio-crítico de la lectura realizar y analizar las estructuras textuales. Existen dos fundamentos para hacer este ejercicio, el primero de ellos es de carácter epistémico y tiene que ver con la importancia de la lingüística textual en el desarrollo y consolidación de diversas disciplinas. El otro, por la relación discursiva en la que se manifiestan las macroestructuras y las superestructuras en diversos textos y la forma como a través de ellas se puede captar una representación social más profunda del discurso, Van Dijk (2000, p. 18) reserva la noción de "macroestructura" para el tema global del texto y habla de "superestructura" para hacer referencia a su forma local. Esta forma global es definida, como si se llevara el análisis sintáctico al plano del texto, mediante un esquema de organización de las categorías.

La interdisciplinariedad presente en la lectura crítica es un fundamento para comprender la tarea tan importante que como maestros de lengua se tiene ante los estudiantes, la sociedad y el futuro. La interacción de los anteriores aspectos conceptuales brinda la columna vertebral para desarrollar en las aulas la comprensión crítica.

\subsection{El contexto, los discursos y las ideologías}

Otro de los conceptos fundamentales en el desarrollo de la comprensión crítica tiene que ver con el contexto. La teoría del contexto explica cómo los participantes de la situación comunicativa son capaces de ordenar y adaptar (la producción y la recepción/ interpretación) el discurso a la situación comunicativa interpersonal-social. Esta aproximación difiere de otros análisis discursivos por su carácter socio-cognitivo, porque trata de formular la interfaz entre las estructuras de las situaciones sociales y "las maneras en que los actores sociales representan mentalmente esas situaciones, de tal manera que sea posible entender cómo esas representaciones pueden influir la producción y la comprensión del discurso" (Van Dijk, 2001, p. 16).

La representación mental de la situación comunicativa se hace con un modelo específico que llamamos modelo del contexto o simplemente contexto. A diferencia de la situación social, "el contexto no es algo externo o visible, o fuera de los participantes, sino algo que construyen como representación mental" (Van Dijk, 2001, p. 17), de ahí la importancia del contexto en el proceso de comprensión crítica de los discursos.

Los modelos del contexto son una manera concreta de los que formamos a lo largo de nuestras vidas, como las prácticas diarias, por ejemplo, desde la mañana cuando nos levantamos y nos damos cuenta de quiénes somos, dónde estamos, qué estamos haciendo, etc. durante todos los actos y eventos que se presentan durante el día hasta que nos acostamos por la noche. Así, mientras estamos conscientes, permanentemente construimos lo que se denomina modelos mentales de la situación en la que nos ubicamos, de nuestra 
realidad inmediata, la de nosotros mismos, de otra gente, del tiempo, del lugar, de los actos, etc. Mucho de esto se hace a través de la comprensión de los discursos que circulan alrededor y a los que estamos expuestos.

Para entender el desarrollo de la comprensión crítica es fundamental reconocer el concepto de ideología, porque su estudio da forma en la actualidad al enfoque sociocrítico de la lectura. Desde esta visión:

La primera suposición es que cualesquiera sean las ideologías, son principalmente algún tipo de "ideas", es decir, son sistemas de creencias. Esto implica, entre otras cosas, que las ideologías, como tales, no comprenden las prácticas ideológicas o las estructuras sociales.(Van Dijk, 2005, p. 10).

Para que exista una ideología es inevitable que sea compartida por un grupo; las ideologías no son individuales, es decir, son representaciones sociales-fundamentales que identifican un conjunto de personas. No obstante, para que estas sean compartidas, es necesario que cumplan con ciertas condiciones tales como: la pertinencia, las actividades, los valores implicados, los intereses, las metas, la posición y los recursos.

No cualquier tipo de representación que identifica un grupo es una ideología, solo pueden serlo las creencias sociales fundamentales, o axiomáticas, las cuales organizan y controlan otras ideas socialmente compartidas. Un rasgo característico de las ideologías consiste en cumplir una función cognoscitiva, al proporcionar coherencia a las creencias de quienes participan de ellas, es decir, con este sistema general de ideas se podrá tomar posición ante situaciones de la vida cotidiana, las cuales pondrán de manifiesto los valores o antivalores que son importantes para el grupo y que los diferencia de otros, así, la desigualdad, la justicia, la discriminación, la libertad, etc. son los fundamentos de las ideologías. De esta manera, las agrupaciones sociales, explican, originan o legitiman sus acciones a través de discursos ideológicos.

\section{Conclusiones}

Como se mencionó al principio del artículo, las problemáticas de los estudiantes, objeto de este estudio, mostraban vacíos bastante importantes en cuanto al proceso lector. En este sentido, al iniciar los cursos académicos se buscó establecer el nivel de comprensión lectora en que se encontraban los estudiantes, para esto se aplicaron dos pruebas de lectura, en la primera se indaga por la comprensión literal, es decir, se buscó que los estudiantes dieran cuenta del contenido textual del documento presentado en la prueba; posteriormente se aplicó otra prueba que buscaba establecer relaciones inferenciales entre la información explícita y la implícita. 
Al evaluar los resultados, se evidenció que los estudiantes alcanzan buenos niveles de comprensión literal, llegan a un 80\% de efectividad, pero los resultados de la segunda prueba ponen de manifiesto que la comprensión inferencial no la logran ni siquiera la mitad de los estudiantes de cada uno de los grupos con los que se estaba trabajando.

Por lo anterior, se hizo necesario planear una intervención pedagógica para lograr que los estudiantes accedieran a un tipo de comprensión superior al nivel inferencial, es decir, a un nivel crítico, pero a la luz de unas categorías de análisis que permitirían aportar no solo al desarrollo de habilidades para la comprensión, sino que además ayudaran en la formación de ciudadanos críticos y reflexivos.

Durante la mayor parte del curso se pretendió que los estudiantes se apropiaran de estrategias para la comprensión de textos que iban desde la identificación del tipo de texto, la elaboración de una macroestructura referencial, para, específicamente, reconocer la o las hipótesis, los argumentos y algunos marcadores textuales; además de establecer las condiciones de producción del discurso.

Al principio se buscó que de manera oral los estudiantes explicaran lo que entendían, que describieran el procedimiento seguido para el análisis y, de esta manera, la clase se convertía por momentos en interesantes debates; luego esa argumentación debía plasmarse de forma escrita y socializarla en el grupo, allí los estudiantes eran co-evaluadores con ayuda, por supuesto, del maestro.

Otra estrategia que contribuyó al desarrollo de la comprensión, fue realizar los ejercicios de lectura con un fin particular, en otras palabras, propusimos una serie de momentos en los que cada ejercicio se convirtió en un insumo para crear un artículo de indagación producto del proceso académico mencionado. Un aspecto novedoso que se desarrolló a lo largo de la investigación, fue proponer la lectura de discursos sociales y políticos, lo que contribuye en la formación de un lector, pero sobre todo de un ciudadano crítico, objetivo fundante de este ejercicio de investigación.

La aproximación preliminar que se ha hecho ante el estudio de la lectura crítica permite inferir la necesidad del cambio en las prácticas de comprensión de los discursos que circulan en el ámbito académico y social de nuestros estudiantes, la problemática no solo se centra en uno de los agentes educativos, por el contrario lo que se buscó fue proponer alternativas que reúnan y propicien una acercamiento al desarrollo de las estrategias cognitivas y meta-cognitivas para comprender críticamente los diferentes textos que circulan en el proceso de formación académica y social. 
Desde el punto de vista metodológico, la investigación permitió legitimar el enfoque cualitativo como elección para el estudio de la lectura crítica, puesto que favoreció el conocimiento de la situación social que rodeaba el análisis de los textos y generó las condiciones adecuadas para que los lectores discutieran sus puntos de vista. Con la elección del enfoque, se desarrolló la comprensión, interpretación e intervención de la problemática y no solo se brindó una explicación del fenómeno.

Al finalizar los cursos donde se efectuó el trabajo investigativo se pudo reconocer algunos avances en la comprensión de diversos textos trabajados durante el semestre; dichos aspectos hacen referencia a la identificación del tipo de texto, la representación esquemática del contenido literal, donde se representan las relaciones del contenido para luego mostrar el significado de manera clara y comprensiva; los ejercicios que implicaron intertextualidad mostraron algunas dificultades, por la falta de costumbre al establecer relaciones entre autores, teorías o conceptos.

A partir del trabajo realizado, se consolidó uno de los semillero de investigación reconocido por la universidad, llamado: "Entre Comillas: Análisis de los Mass media, una visión de la lectura crítica", cuyo objetivo central se ha encaminado al desarrollo de procesos de comprensión crítica en las diferentes realidades discursivas de los mass media. Asimismo, la investigación contribuyó a las reflexiones sobre la lectura como práctica social y responde a las políticas educativas que buscan la formación de ciudadanos reflexivos, autónomos y con capacidad crítica.

\section{Referencias bibliográficas}

Atorresi, A. y Ravela, P. (2009). Los proyectos de evaluación formativa y auténtica. Montevideo, Instituto de Investigaciones en Evaluación, Montevideo: Universidad Católica del Uruguay.

Bruner, J. S. (1984). Acción, pensamiento y lenguaje. Madrid: Alianza Editorial

Camps, A. \& Colomer, T. (1996). Enseñar a leer, enseñar a comprender. Madrid: Celeste.

Cassany, D. (2006). Tras las líneas. Sobre la lectura contemporánea. Barcelona: Anagrama.

Cassany, D. (2005). Los significados de la comprensión crítica. Revista Lectura y Vida, 26(3), 32 45. Buenos Aires: Universidad Nacional de La Plata. Recuperado de http://www.lecturayvida. fahce.unlp.edu.ar/numeros/a26 n3/26_03_Cassany.pdf 
Moreno, J. et al. (2010). Prácticas lectoras: comprensión y evaluación. Tendencias, estado y proyecciones. Forma y Función, 23(1), 145-175. Bogotá: Universidad Nacional de Colombia. Recuperado de http://www.redalyc.org/articulo.oa?id=21916690009

Pardo, N. (2008). ¿Qué nos dicen? ¿Qué vemos? ¿Qué es...pobreza? Análisis crítico de los medios. Bogotá: Universidad Nacional de Colombia.

Santiago, W. (2010). Lectura, Metacognicion y evaluación. Bogotá: Alejandría, Libros.

Serrano, M. (2007). Teoría de la Comunicación. La comunicación, la vida y la Sociedad. -Hill, Madrid: Mac Graw.

Smith, F., (1983). Comprensión de la lectura, análisis psicolingüístico de la lectura y su aprendizaje. México: Trillas.

Unesco. (2009). Segundo estudio regional comparativo y explicativo. Aportes para la enseñanza de la lectura. Santiago, Chile: Salesianos Impresores S.A.

Van Dijk, T. (2001). Algunos principios de una teoría del contexto. Recuperado de: http:// material.producciondetextos.com.ar/2012_bib_21_van_dijk_algunos_principios_ teoria_contexto.pdf.

Van Dijk, T. (2003). De La Gramática del Texto al Análisis Crítico del Discurso. Recuperado de: http://www.discourse-in-society.org-/teun.html.

Van Dijk, T. (2004). Discurso y Dominación. Recuperado de: http://www.bajofuego.org.ar/textos/ Discurso_y_dominacion.pdf.

Van Dijk, T. (2005). Ideología y análisis del discurso. Recuperado de: http://www.scielo.org.ve/ scielo.php?pid=S131552162005000200002\&script=sci_arttext.

Van Dijk, T. (Comp.) (2000). El discurso como estructura y proceso. Gedisa. Barcelona.

Wodak, R. y Meyer, M. (2001). Métodos de análisis crítico del discurso (Trad. Tomás Fernández Aún y Beatriz Engibar, 2003). Barcelona: Gedisa. 